\title{
Development of Non-Contact Detection of a Charged Conductor and Visualization System
}

\author{
Kazuma Nogi ${ }^{1}$, Yoshihiko Kuwahara ${ }^{1 *}$, Takuji Ushimoto \\ ${ }^{1}$ Department of Electric and Electronical Engineering, Faculty of Engineering, Shizuoka University, Hamamatsu, Japan \\ ${ }^{2}$ Division of Research and Development, Chubu Electric Power Co., Inc., Nagoya, Japan \\ Email: *kuwahara.yoshihiko@shizuoka.ac.jp
}

How to cite this paper: Nogi, K., Kuwahara, Y. and Ushimoto, T. (2021) Development of Non-Contact Detection of a Charged Conductor and Visualization System. Open Journal of Safety Science and Technology, 11, 12-26.

https://doi.org/10.4236/ojsst.2021.111002

Received: February 3, 2021

Accepted: March 22, 2021

Published: March 25, 2021

Copyright $\odot 2021$ by author(s) and Scientific Research Publishing Inc. This work is licensed under the Creative Commons Attribution International License (CC BY 4.0).

http://creativecommons.org/licenses/by/4.0/

\begin{abstract}
At a substation, the condition of an applied voltage is checked using a bulky contact-type electroscope. Although it is important to secure a safe working environment, it is a burden to workers. In addition, in an emergency investigation or at the time of an accident, an operator may erroneously recognize a power interruption and cause an electric shock. Therefore, it is desirable to develop technology that can easily distinguish the charged condition from a distance. In this research, the focus was on the slight vibration of the conductor owing to the Coulomb force generated when a high voltage was applied. A system for visualizing the charging state by detecting the vibration with radar and combining it with a stereo camera was developed. Its performance was then confirmed at an experimental site and substation. In addition, the effectiveness of a method that used independent component analysis to identify the harmonics of the power supply frequency and vibration resulting from the applied voltage was demonstrated.
\end{abstract}

\section{Keywords}

Electric Power, Electroscope, Millimeter-Wave Radar, Visualization, Stereo Camera, Independent Component Analysis

\section{Introduction}

At workplaces that handle high voltage (several to several tens of kilovolts), such as substations, work should be carried out after confirming that the electric circuit is in a power outage state to avoid electric shock accidents. However, in an emergency investigation or disaster, the operator may misidentify the power outage and cause an electric shock that may lead to an accident. Furthermore, if there are many inspection points, much time is needed to check the power out- 
age status, and the work efficiency is poor. Therefore, it is desirable to develop a technology that can easily confirm the power outage state without contact.

We focused on the fact that when a high voltage was applied to a conductor, a slight vibration is generated by the Coulomb force [1]. Using the millimeter wave radar, it was confirmed that the frequency can be estimated by irradiating the charged conductor with continuous wave $(\mathrm{CW})$ and receiving, and processing the reflected wave. Furthermore, we proposed a method to determine the power applied status by separating and identifying two parallel wires in the beam [2]. However, separation and identification were difficult in an environment with many reflections such as inside a substation. Therefore, we proposed a method to narrow the beamwidth to detect only one electric wire, and identify the intended wire by the distance estimation result combining the frequency modulation continuous wave (FMCW) and a stereo camera [3] [4]. Using these technologies, we developed a system that visualizes the applied power state of conductors and evaluated its performance under various conditions [5]. If the target cannot be seen, or if the return signal cannot be captured stably due to the influence of reflection by the surrounding structures, operator needs to use it closer to the target. Our users allow us to measure as close as $1 \mathrm{~m}$ to the target.

In the actual measurement environment, the vibration caused by the charged electric wire and the vibration including the harmonic component of the surrounding power supply device is mixed. Therefore, it is necessary to separate and identify these components. In this paper, a method of separating the vibration component by the charged electric wire and other components is proposed by applying the independent component analysis [6]. We will also present the operating principle of the system, the outline of the prototype device, and the performance evaluation experiment.

\section{Principle of Vibration Detection and Distance Estimation}

\subsection{Vibration and Distance Estimation by Millimeter Wave Radar}

As shown in Figure 1, the conductor vibrates at a frequency twice the applied voltage with attractive and repulsive forces of magnitudes corresponding to the voltage and current owing to the Coulomb force generated inside the conductor and the electromagnetic force between the conductors in the vicinity. For example, in the case of a conductor that is charged with a frequency of 60 $\mathrm{Hz}$, which is the frequency of commercial power sources in western Japan, attractive and repulsive forces are generated at the peaks and valleys of the waveform, and the conductor vibrates at $120 \mathrm{~Hz}$. The vibration can be detected by irradiating the conductor in this state with radar radio waves. In the case of the above-mentioned commercial power supply example, $120 \mathrm{~Hz}$, which is a frequency of the vibration, is superimposed on the received wave. Therefore, by multiplying the transmitted wave by the mixer at the receiver and passing it through the low frequency pass filter, a sine wave of $120 \mathrm{~Hz}$ is obtained. At this time, the distance to the conductor can be estimated using the FMCW radar. In 


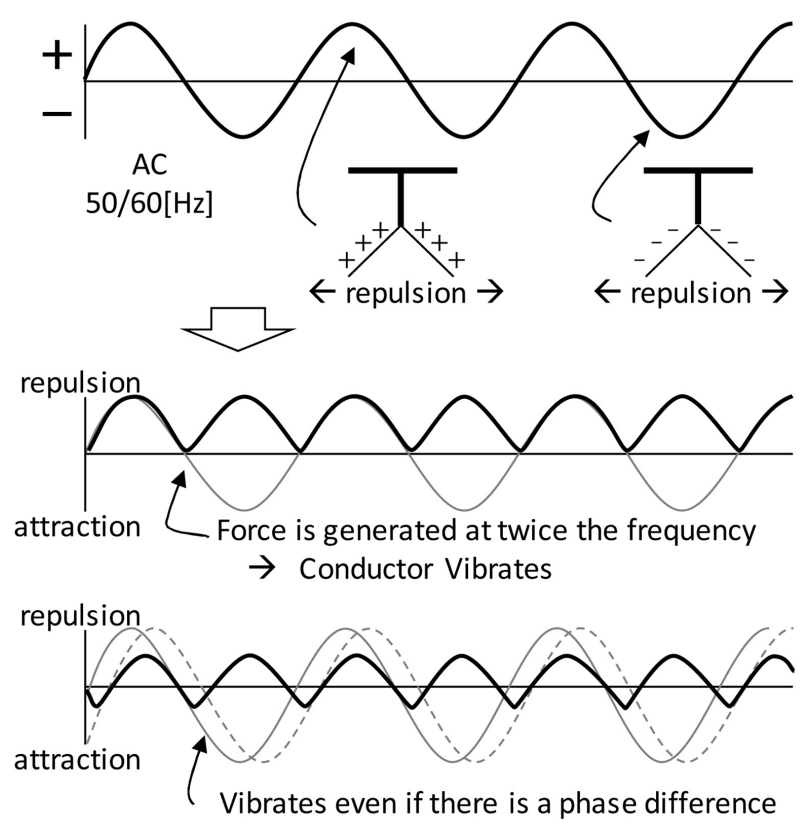

Figure 1. Principle of detecting vibration.

the FMCW mode, the transmission frequency is changed in a saw-like manner, and the distance to the reflector can be measured from the beat frequency in the received wave.

\subsection{Identifying Conductors and Measuring Distance with a Stereo Camera}

Distance estimation by FMCW radar alone does not indicate whether the target captured by the antenna is the conductor intended by the operator. By identifying the conductor with a stereo camera and measuring the distance, the conductor captured by the radar and the conductor intended by the operator can be matched.

When measuring the distance with a stereo camera and displaying the result of the power charge judgment, the target conductor must be separated from the background. Therefore, image processing to identify the target conductor is utilized. To separate and extract a specific object on an image, there are known methods such as filtering by a specific color and brightness, and edge detection that finds a change point in the image by differentiation. Because the conductor is a single color and there is little change in color within the same object, the conductor can be extracted by the red-green-blue value.

There are various methods for estimating the distance using multiple cameras, but this unit uses the method using a stereo camera, which is relatively easy to prepare for placement and calibration [7]. With a stereo camera, an object is photographed by two cameras placed in parallel, similar to the human eye, and the distance from the camera is determined by the principle of triangulation using the parallax at the same object point on the screen. As shown in Figure 2, assuming that the depth from the camera to the target is $Z$, the focal length of 


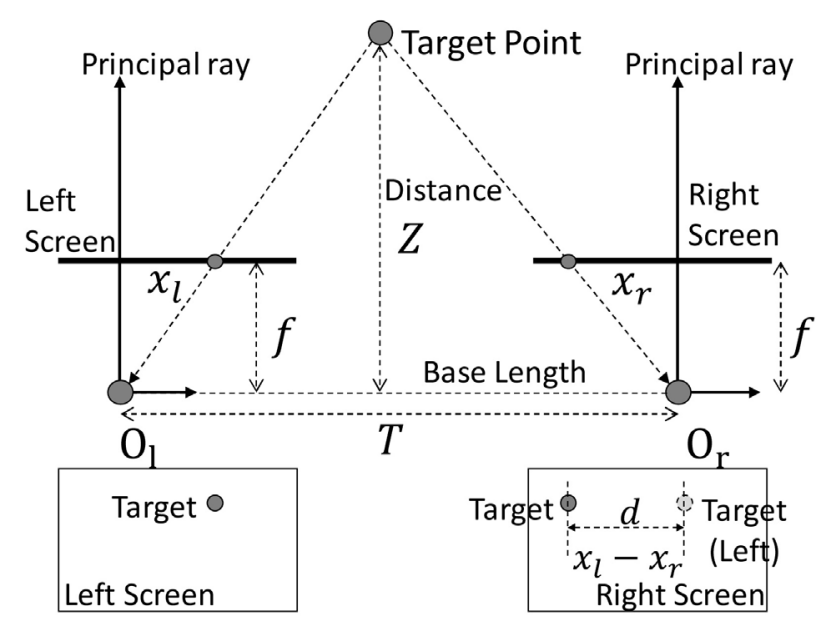

Figure 2. Principle of measuring distance by stereo camera.

the camera is $\mathrm{f}$, the distance between the cameras is $T$, and the position pixels of the target points taken by the left and right cameras are $x_{I}$ and $x_{I}$, respectively, the distance is calculated by the following equation.

$$
Z=\frac{f T}{x_{l}-x_{r}}
$$

The left and right cameras were arranged so that their line of sight coincided with the irradiation center of the radar.

\section{System Design}

\subsection{Hardware}

This machine consists of the units shown in Table 1. Figure 3 shows the hardware configuration and appearance of the prototype device.

To realize the radar function, the evaluation board EV-RADAR-MMIC2 [8] of MMIC for in-vehicle millimeter-wave radar manufactured by Analog Devices, Inc. was used. This evaluation board is equipped with a two-channel transmission integrated circuit (IC) ADF5901, a four-channel reception IC ADF5904, and a modulation/demodulation IC ADF4159, each of which can be controlled by serial peripheral interface (SPI) communication. In this research, a board that converts SPI and Universal Serial Bus (USB) signals was added to this, and such radar operation as range measurement could be controlled with a single USB port.

To detect vibration, video signal of the board sends to DAQ USB-6001 manufactured by National Instruments. In order to match the voltage range of the video signal on the board $(-4 \mathrm{~V}$ to $4 \mathrm{~V})$ to the input range of DAQ $(-10 \mathrm{~V}$ to $10 \mathrm{~V}$ ), a video amplifier with an amplification factor of 2.5 was inserted between the video output and DAQ. The video output of the radar unit collects data for 1 second at a sampling frequency of $20 \mathrm{kHz}$ and converts it into a digital signal. In vibration detection, an antialiasing filter is not required since the maximum frequency of the observed signal is $300 \mathrm{~Hz}$. 
Table 1. Basic components of visualization system.

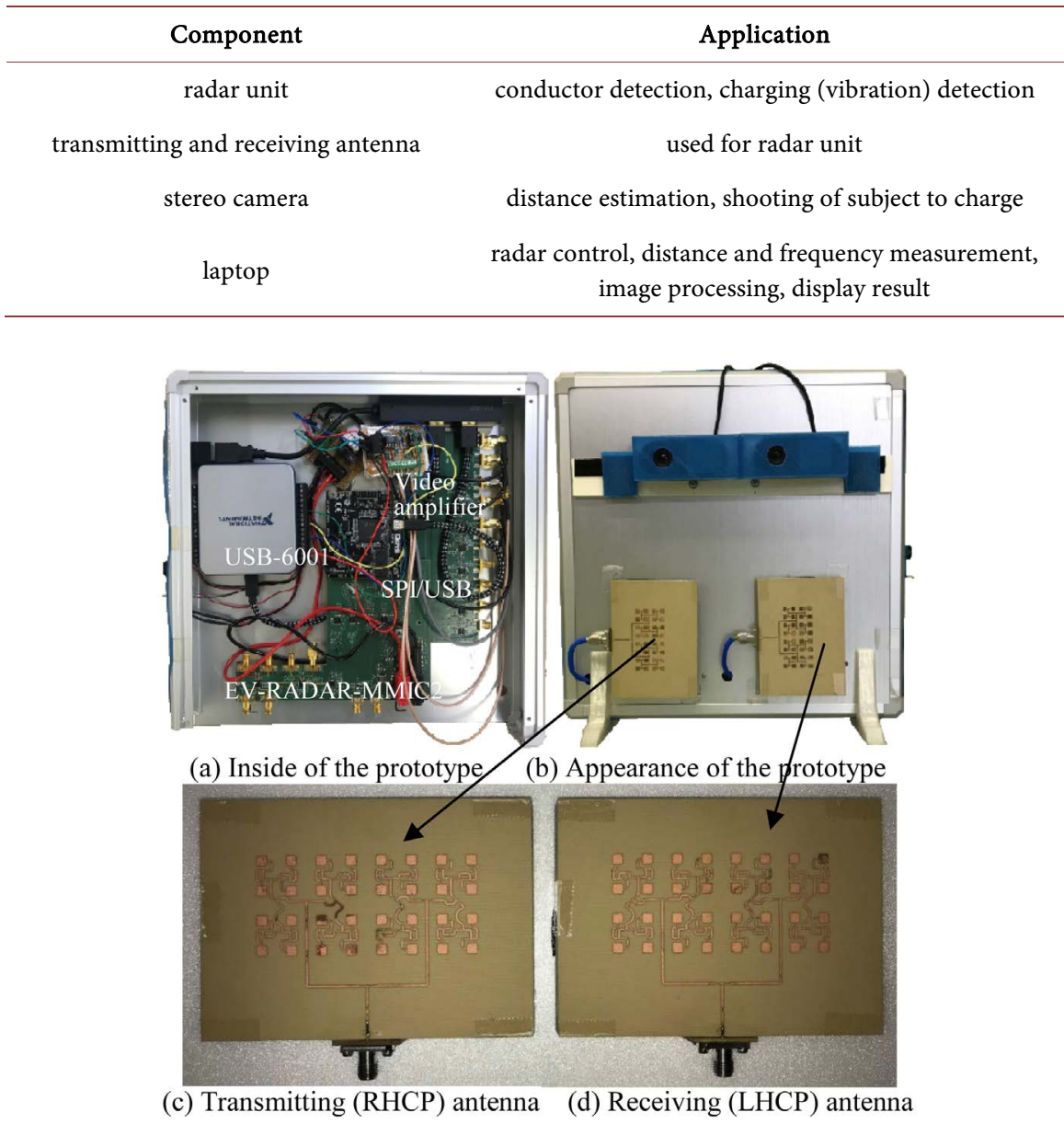

Figure 3. The prototype device.

As shown in Figure 3(c) and Figure 3(d), the antenna was a 32-element planar patch antenna array in the $24 \mathrm{GHz}$ band, and it was designed with circularly polarized waves. Because the conductor has an elongated shape, linearly polarized waves are advantageous in terms of received power, but it is necessary to align the plane of polarization with the target. It may lead to operational inconvenience. When the metal is irradiated with a circularly polarized wave, the direction of rotation is opposite. A right-handed circularly polarized antenna was used for transmission and a left-handed circularly polarized antenna was used for reception. Circularly polarized waves were generated using a sequential array. The direction of rotation of polarized waves was changed by changing the direction of the Single fed CP patch and the feeding position [9].

Table 2 and Table 3 show the main specifications and antenna design requirements, respectively. The maximum measure distance of this system was set to $6 \mathrm{~m}$ due to the performance of commercial radar units. The beam field of view at a distance of $6 \mathrm{~m}$ was limited to $2 \mathrm{~m} \times 1 \mathrm{~m}$ to prevent multiple wires from entering the radar field of view during outdoor operation. The size of this field of view was determined empirically. When the two wires enter the field of view, the 
Table 2. Main specifications.

\begin{tabular}{cc}
\hline Performance index & Target value \\
\hline Irradiation range to the conductor $3 \mathrm{~m}$ ahead & $1.0 \times 0.5 \mathrm{~m}^{2}$ \\
Maximum detection distance & $\geq 6 \mathrm{~m}$ \\
Transmission/reception isolation & $\geq 60 \mathrm{~dB}$ \\
\hline
\end{tabular}

Table 3. Design requirements and evaluation results for antenna.

\begin{tabular}{ccc}
\hline Parameters & Target value & Evaluation results \\
\hline Beam width & $20 \times 10 \mathrm{deg}$ & $20 \times 10 \mathrm{deg}$ \\
Gain (circularly polarized wave) & $>20 \mathrm{dBi}$ & $18.4 \mathrm{dBi}$ \\
Axial ratio & $<1.0 \mathrm{~dB}$ & $2.15 \mathrm{~dB}$ \\
$\begin{array}{c}\text { Distance between transmitting and } \\
\text { receiving antennas } \\
\text { Sidelobe level }\end{array}$ & $>40 \mathrm{~mm}$ & $85 \mathrm{~mm}$ \\
& $<-13 \mathrm{~dB}$ & $-13.4 \mathrm{~dB}$ \\
\hline
\end{tabular}

operator either moves the field of view or approaches the wires and narrows the field of view for measurement.

To obtain the performance shown in Table 2, the shape of the antenna was optimized by combining the genetic algorithm and the trust region method [11]. The power divider is formed on the same surface as the patches. The impedance of the array antenna is $50 \mathrm{ohms}$ and the measured VSWR is 1.92. The evaluation results of the antenna are also shown in Table 3.

A USB-connected Web camera C270 was used as the stereo camera. This camera is inexpensive and easily available. Although it is capable of shooting 30 -fps movies with 720p HD image quality, it is compact and lightweight, so it meets the specifications sufficient for this distance estimation application.

The evaluation boards, USB DAQ, power supply, and amplifier were put together in an aluminum case. The side was equipped with a USB port as an interface, a DC5V input terminal and a power switch. A stereo camera and a mounter for a transmission/reception antenna were provided on the front.

\subsection{Software}

For data recording from radar, frequency analysis, image processing and display, a program was created using LabVIEW (National Instruments) because of its ease of connection with external devices and portability. In LabVIEW, utilities for DAQ and functions for image acquisition are prepared in advance, libraries created in different programming languages can be easily called, and graphical user interface programs can be performed efficiently.

Figure 4 shows the operation flow. When the camera is held so that the conductor to be detected is in the center, the system recognizes the range of the conductor and measures the distance to the conductor using a stereo camera image (camera processing). Subsequently, the radar unit operates in the FMCW mode and measures the distance to the conductor based on the reflection time of 


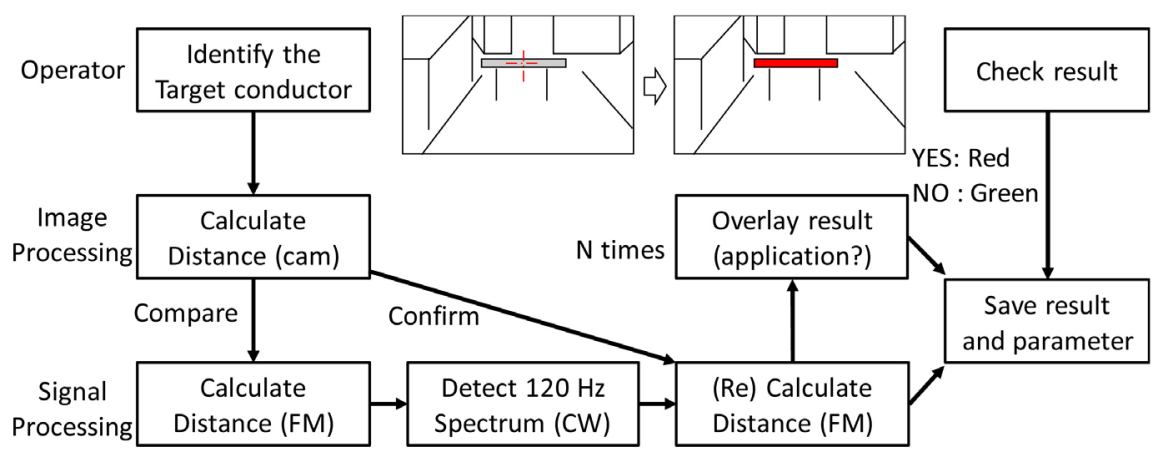

Figure 4. Design requirements and evaluation results for antenna.

radio waves. After comparing the two measured values and confirming the match, the radio wave irradiation range is calculated from the distance to the conductor and the display range is adjusted so that the user can confirm whether the device correctly recognizes the measurement target. At this time, switching to the vibration estimation mode by $\mathrm{CW}$ becomes effective. In vibration detection in CW mode, measurement is performed multiple times. If the spectrum at the target frequency is recognized a certain number of times, it is judged that the conductor is vibrating. Then, it switches to the FMCW mode and stereo camera again. If the distance and image have not changed significantly, the power charge status is displayed on the screen.

\section{Experiment}

To verify the performance of the prototype system, a preliminary experiment of distance measurement and vibration detection was conducted in a corridor on campus. Power charge detection in the substation equipment was also evaluated. In the experiment, a measurement assist device that combines two stepping motors was used so that the irradiation range of the radar could be accurately adjusted and evaluated. As shown in Figure 5, the measurement assist device consisted of Motor1, which controls the elevation angle, and Motor2 which controls the azimuth angle.

After the above operation verification, the voltage was changed in the range of 2 to $20 \mathrm{kV}$ at the high-voltage experimental facility of the Electric Power Technology Laboratory of Chubu Electric Power Co., Inc., and charge detection using only the voltage was evaluated. Then, in an actual substation, a field experiment of power charge detection was conducted using the proposed system and the overall performance was evaluated. The evaluation results are reported below with typical examples of experimental results.

\subsection{On-Campus Experiment}

\subsubsection{Operation Check}

As a preliminary experiment, the functioning of distance and vibration detection was confirmed using the panel heater and copper bar shown in Figure 6 for a reflector and a speaker for the vibration source. 

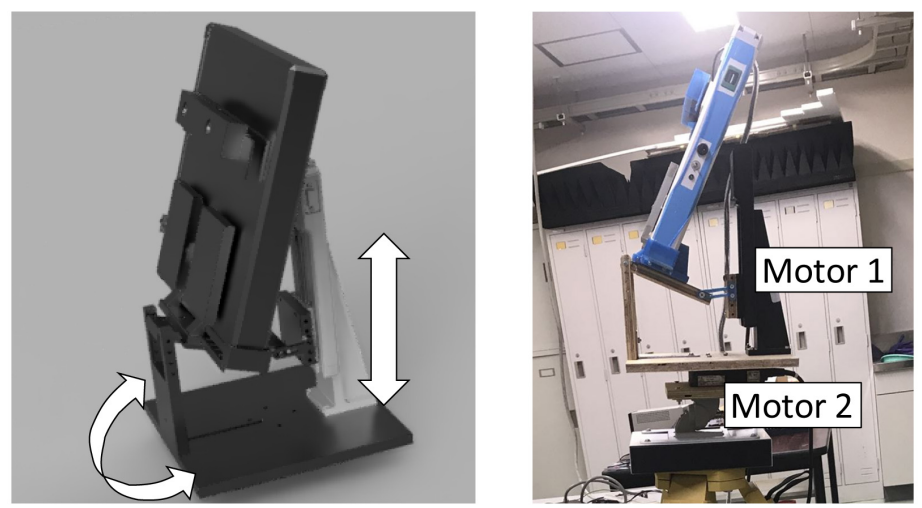

Figure 5. Measurement assist device.

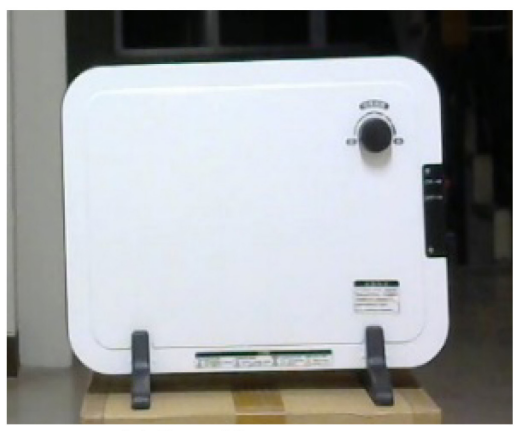

(a) Panel heater

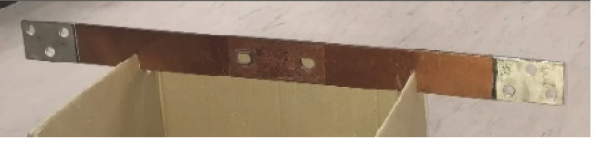

(b) Copper bar

Figure 6. Reflector.

\subsubsection{Identification of the Object}

Figure 7 shows the state of the preliminary experiment for distance measurement. This figure shows that the measurement result of the distance to the target is $1.5 \mathrm{~m}$ for both the stereo camera and FMCW radar. The object identified by the camera is filled with blue. In addition, the measurement result by the radar is shown by the peak of the graph. The vertical field of view is indicated by a green belt, and the objects collated by the camera and radar are indicated by brown.

\subsubsection{Power Charge Detection}

Because it was possible to confirm identification of the wire, the power charge detection was evaluated for the $100 \mathrm{~V}$ bus at the power receiving equipment on campus, a current of 120 A flowed in the bus. Representative results are shown in Figure 8. The red circle in the photograph is the irradiation center of the beam. As shown in the upper figure of Figure 8, when the prototype device was placed facing the position of the bus that was not bolted and the measurement was performed, the received wave contained a component of $120 \mathrm{~Hz}$. The part highlighted in red in the figure is the power-charged part detected by image processing. The spectrum of $120 \mathrm{~Hz}$ disappeared when the line of sight was removed from the bus by turning the beam direction to the left or right or changing the height. In addition, it was difficult to detect vibration when the beam was irradiated to the fixed part as shown in the lower figure of Figure 8. 


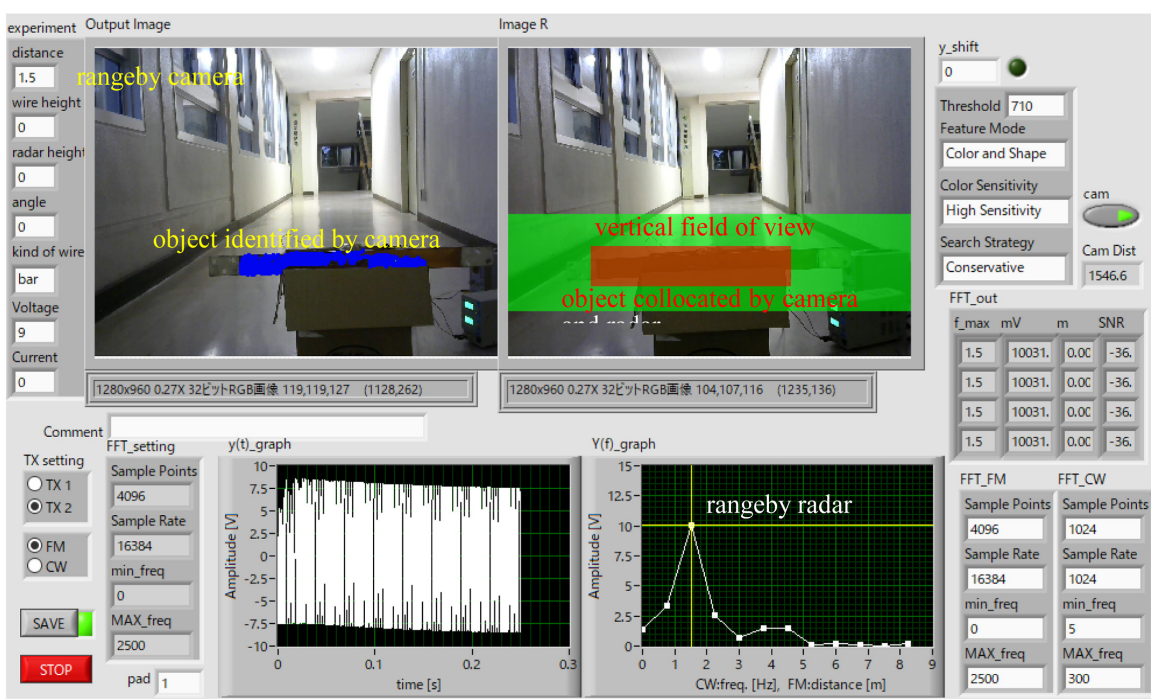

Figure 7. Evaluation of range measurement.
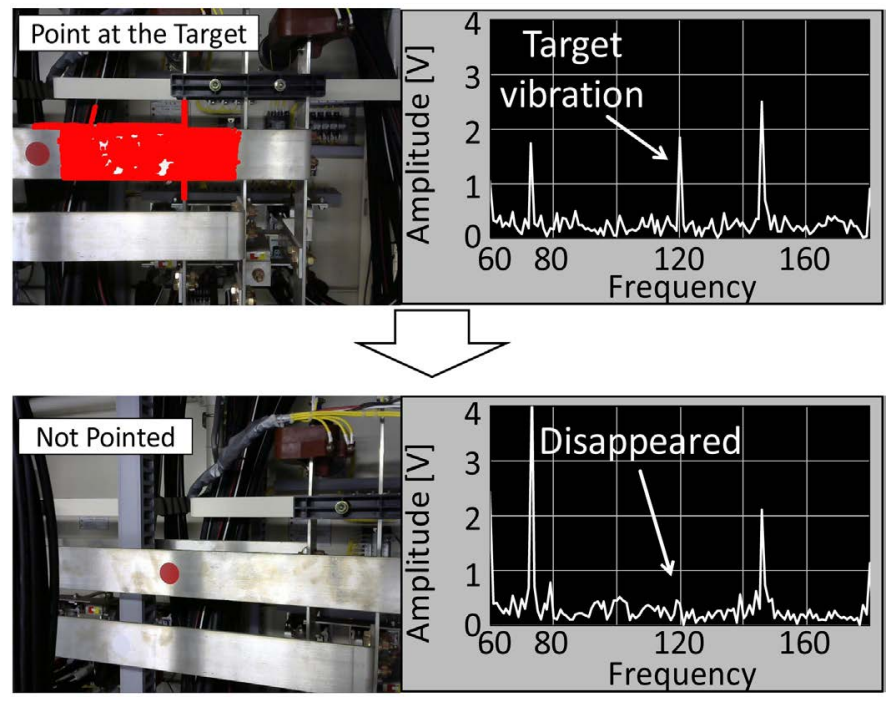

Figure 8. Verification of voltage detection at substation facilities $(100 \mathrm{~V}, 120 \mathrm{~A})$.

\subsection{Detection only by Charging}

\subsubsection{Experimental Setup}

To investigate whether power application can be detected with a conductor in which a high voltage is applied but no current is flowing (this situation can occur because of maintenance/inspection of electric power equipment, etc.), verification was performed using the experimental equipment in the high-voltage experimental equipment of the Electric Power Technology Laboratory in Chubu Electric Power Co., Ltd.

We applied 2 to $20 \mathrm{kV}$ to copper rods, twisted wires, and pipes, and investigated the intensity of the $120 \mathrm{~Hz}$ spectrum contained in the received signal at a measurement distance of 1.0 to $2.0 \mathrm{~m}$. Figure 9 shows the state of the experiment and measurement target. 


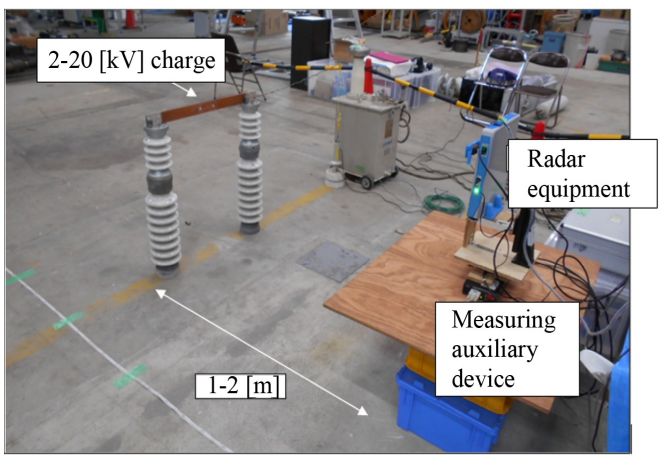

(a) Scenario of experiment

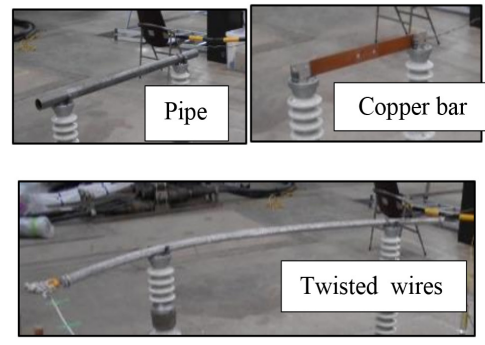

(b) Object

Figure 9. Detection evaluation only by charging.

\subsubsection{Experiment}

Figure 10 shows the relative intensity of the $120 \mathrm{~Hz}$ spectrum when the measurement distance is $1 \mathrm{~m}$ and a circularly polarized antenna is used. The copper bar has the largest spectrum at $120 \mathrm{~Hz}$. Because the pipe is curved, only part of the reflected wave can be received by the radar, and the lobing phenomenon occurs because of interference from ground reflection. In this case, it is necessary to take such measures as increasing the height to be measured.

At the time of these measurements, a spectrum was also observed at $60 \mathrm{~Hz}$. The amount of change in the $60-\mathrm{Hz}$ spectrum with respect to the applied voltage was approximately half that of $120 \mathrm{~Hz}$, and no significant change was observed depending on the type of conductor. Therefore, the vibration of $60 \mathrm{~Hz}$ was probably caused by the housing of the high voltage equipment.

The change in the $120 \mathrm{~Hz}$ spectral intensity was investigated for the copper bar by changing the charging voltage and distance. A vibration of $120 \mathrm{~Hz}$ could be detected by applying a power of $4 \mathrm{kV}$ or more at $1.0 \mathrm{~m}$ and $10 \mathrm{kV}$ or more at $2.0 \mathrm{~m}$. Below these voltages, the spectrum of $120 \mathrm{~Hz}$ was buried in noise, and the spectrum could not be confirmed by a simple fast Fourier transform (FFT) calculation, so it was judged to be in a power failure state.

\subsection{Field Evaluation at Substations}

We have verified the power charge detection inside and outside an operating sub-station.

\subsubsection{Indoor Experiment}

In the indoor verification, the distance between the antenna and the bus (copper bar) was approximately $1.5 \mathrm{~m}$. Figure 11 shows the measured copper bar and the measurement results of distance and vibration. In the graph of the distance measurement result, a peak is seen at the position of $6 \mathrm{~m}$, which results from the reflection of the wall of the building. The intensity of the detected $120 \mathrm{~Hz} \mathrm{spec-}$ trum was small despite the presence of current. It is probable that the copper bar was fixed firmly and the conductor had a structure that was less likely to vibrate than in the above-mentioned experiment with only power application. 


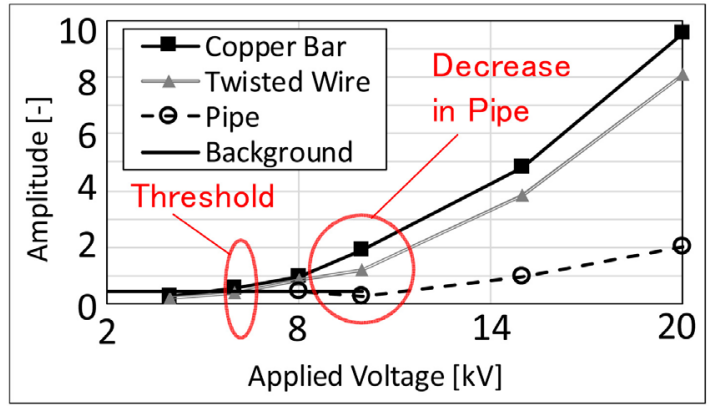

Figure 10. $120 \mathrm{~Hz}$ spectral intensity for each target.

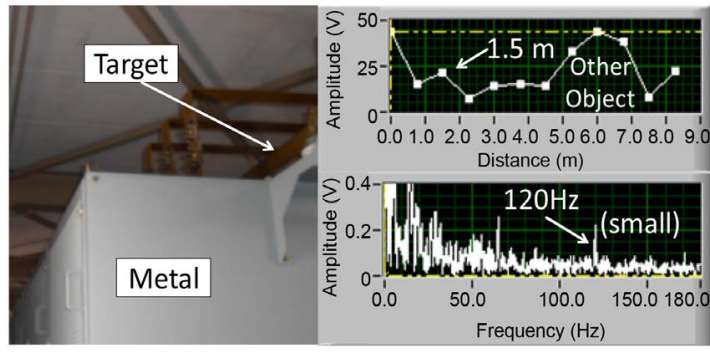

Figure 11. Detection of electricity applied to indoor substation $(6.6 \mathrm{kV})$.

\subsubsection{Outdoor Experiment}

In the outdoor verification, conductors of two different diameters located approximately $4 \mathrm{~m}$ above the ground were measured. The applied voltage was 44.5 $\mathrm{kV}$, and the distances to the conductor were approximately $3.0 \mathrm{~m}$ and $5.0 \mathrm{~m}$. Figure 12 shows the results of distance estimation and vibration detection between the thin and thick conductors to be measured.

With thin conductors, sufficient reflected power was not obtained for distance measurement and vibration detection. Although it was necessary to shorten further the distance between the conductor and the measuring device for detection, it was not possible to measure at a position closer than $3.0 \mathrm{~m}$ because of safety restrictions. In addition, the device shown in Figure 3 was attached to a telescopic pole with a height of $5 \mathrm{~m}$ for measurement, resulting in loss of stability, and the $120 \mathrm{~Hz}$ spectrum of the target was sometimes buried by vibration caused by the wind.

For a thick conductor, distance measurement and vibration detection were possible as long as the wind did not shake the measuring device.

\section{Improvement of the Ability to Detect Power Charges}

In the above-mentioned experiments, the FFT calculation was applied when displaying the received spectrum. If the number of samples increases over time, the frequency resolution increases, the noise components are averaged, and the signal-to-noise-ratio improves. In the experiment, the realistic signal acquisition time was basically $1 \mathrm{~s}$, and 3 to $5 \mathrm{~s}$ at the longest. The $120 \mathrm{~Hz}$ spectrum is very small and does not appear to exist when the object to be measured is tightly fixed 

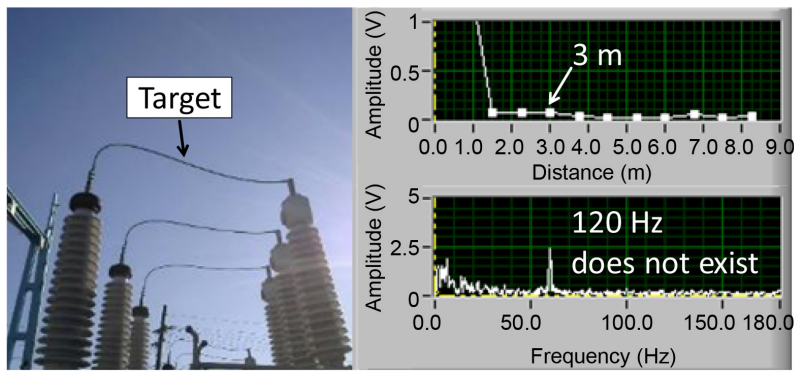

(a) Measurement results for thin conductors
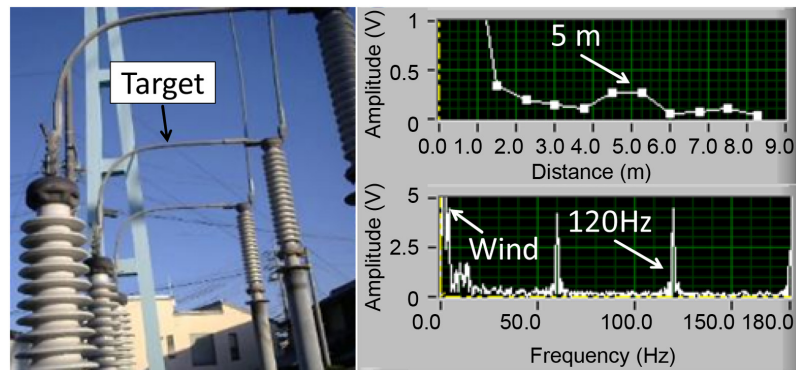

(b) Measurement results for thick conductors

Figure 12. Detection of electricity applied to outdoor substation $(44.5 \mathrm{kV})$.

or the applied voltage is small.

In addition, since various devices are installed in the substation, and vibrations of $60 \mathrm{~Hz}$ and its harmonics can be observed from these devices. The insulator that holds the electric wire may also vibrate due to the vibration of the installation stand even though the electric wire is not charged. For this reason, it is necessary to separate and identify the vibration from the target power-charged wire. Here, assuming that the vibration caused by the charged power is independent of the surrounding vibration source, an attempt was made to separate the components using ICA.

\subsection{Independent Component Analysis}

ICA is one of the analysis methods for multidimensional signals. It is a method for separating signals from linearly mixed signals into independent components using higher-order statistics. It is widely applied in various fields, such as biological signals (e.g., brain waves, heartbeat and respiration), and the vibration analysis of vehicles. In ICA, it is assumed that statistically independent signals (i.e., original signals) $s(t)$ are linearly combined by the mixed matrix $A$, as shown in Equation (2).

$$
x(t)=A s(t)
$$

By multiplying the observed signal $x(t)$ by the inverse matrix $A^{-1}=W$ of the mixed matrix, the signal $\hat{s}(t)$ that seems to be the original signal is obtained.

$$
\hat{s}(t)=W x(t)
$$

Because only the observed signal is known in the real environment, the original 


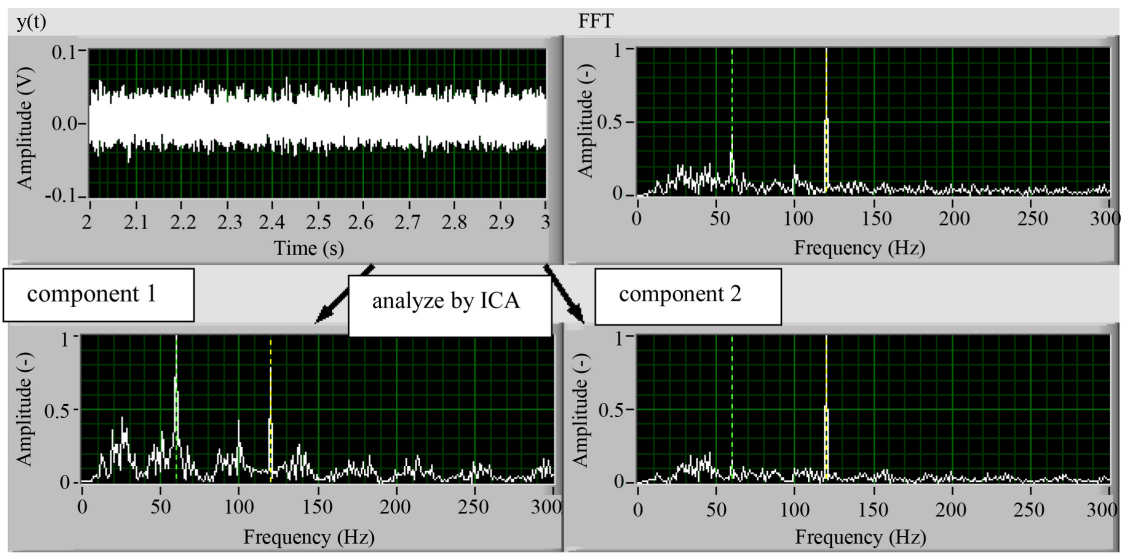

(a) $20 \mathrm{kV}$ electricity application

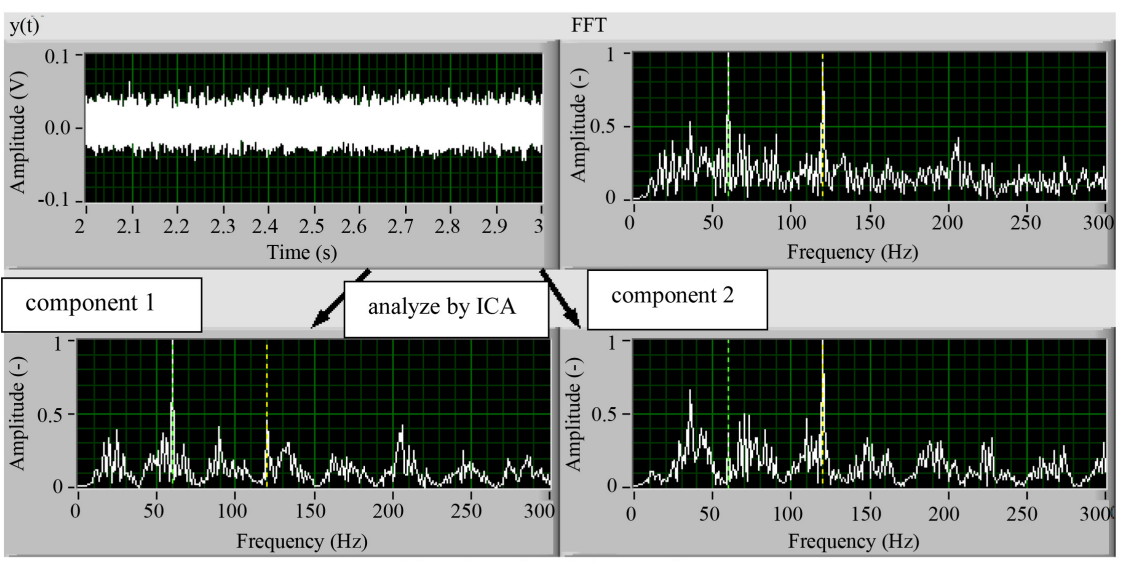

(b) $10 \mathrm{kV}$ electricity application

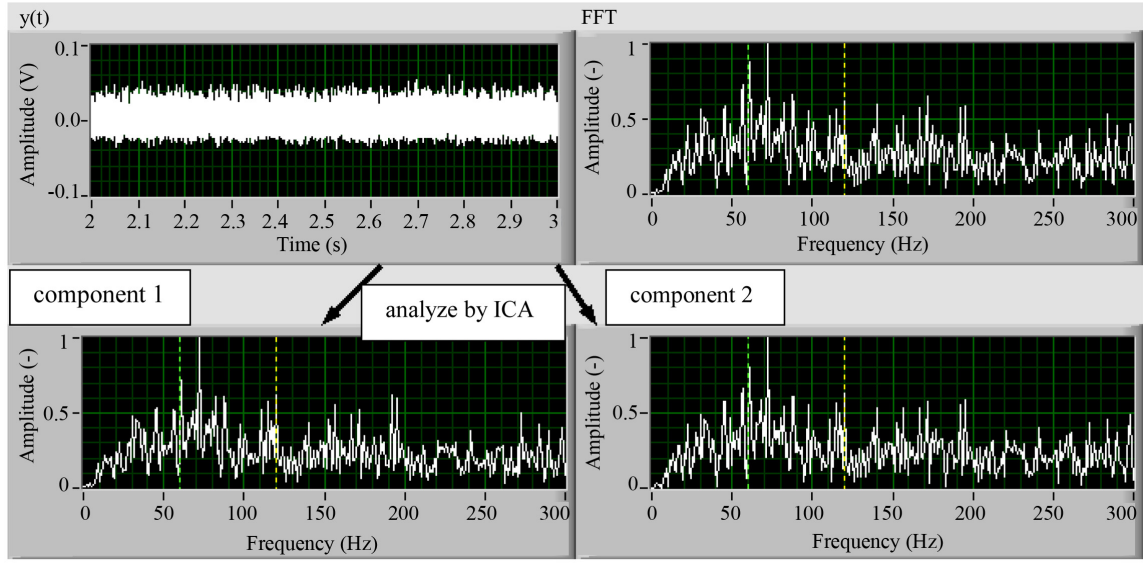

(c) No electricity

Figure 13. Extraction of charge components by ICA.

signal is generated by evaluating $W$ on a statistical scale and updating it sequentially. Here, we try to separate the vibration of the conductor due to Coulomb force from unnecessary signals such as second harmonics generated from the device itself and surrounding devices, air conditioning, outdoor wind, and camera shake.

In the software implementation, FastICA [12], which is a fast and reliable convergence algorithm, was used. In FastICA, the original signal is considered to 
be far from the Gaussian distribution, and the negentropy of the difference between the Gaussian distribution and the target entropy is used as a measure of non-Gaussianness. The separation matrix $W$ is obtained by performing iterative calculations using the fixed point method to maximize the negentropy.

In FastICA processing, the received wave of the $\mathrm{CW}$ radar is first input as a mixed signal. Centering to make the average value of the data 0 , eigenvalue analysis, etc. are performed, and the data are whitened as preprocessing. Next, $W$ is initialized to 0 and repeated until it converges by the fixed-point method. The finally obtained $W$ is used to output the separation signal, and FFT is applied to each to obtain the frequency distribution.

The processing time in ICA was approximately $30 \mathrm{~ms}$ even if FFT was performed after processing ICA for the acquired data for $1 \mathrm{~s}$. In ICA, two observation signals are required to decompose into two signals. Therefore, the signal obtained by shifting the observed signal by $25 \mathrm{~ms}$ was used as another signal [13]. This shift was determined experimentally.

\subsection{Experiments}

Of the measurement data for pipes at a distance of $1.5 \mathrm{~m}$, the separation experiment was performed using the data when the power was applied at $20 \mathrm{kV}$ and 10 $\mathrm{kV}$ and when the power was stopped. The results are shown in Figure 13.

In the $20 \mathrm{kV}$ and $10 \mathrm{kV}$ separation results, the 60 and $120 \mathrm{~Hz}$ spectra have peaks, respectively, and the separation is as expected. The graph with the peak at $60 \mathrm{~Hz}$ on the left side also has a spectrum of $120 \mathrm{~Hz}$, which can be explained as the second harmonic component of $60 \mathrm{~Hz}$. In the graph on the right, the $120 \mathrm{~Hz}$ spectrum is larger than $60 \mathrm{~Hz}$, so it can be judged that the power is being applied. Furthermore, when the power supply was stopped, the spectra after separation were similar, and no spectrum protruding at 60 or $120 \mathrm{~Hz}$ was observed. Therefore, the judgment of the power supply stop could be made correctly. From the above, it is very effective to perform ICA before frequency analysis for power charge determination.

\section{Conclusion}

To detect the charged state of the conductor in a non-contact manner, a voltage detection system that combines a $24 \mathrm{GHz}$ band millimeter-wave radar and a stereo camera was proposed, and its operation was verified using a prototype device. By comparing the distance estimation results obtained by the FMCW radar and the stereo camera in real-time, the identification of the target conductor was confirmed. After verification of the operation in our campus, verification was also performed in a high-voltage power application environment. It was confirmed that a bar-shaped conductor with a large reflection area can be clearly judged to be charged even at a distance of $2.0 \mathrm{~m}$. Even outdoors, if the reflection cross-section of the target is sufficient, the power application judgment can be confirmed, even from a long distance. However, vibration may not be sufficient- 
ly detected when the reflected cross-section of the object is small, when it is not facing directly, or when there is such a disturbance as a strong wind. It was shown that the vibration component resulting from power application and the harmonic component at $60 \mathrm{~Hz}$ generated from the surrounding equipment can be separated by applying ICA as a pretreatment to detect the power application status more reliably. In the final product, we are considering a system in which this system is downsized and mounted on a helmet, and the presence or absence of electric charge is superimposed on the electric wire displayed on the goggles. In the future, we plan to downsize this system, attach it to a helmet, and develop a system that superimposes information of the presence or absence of electric charge on the electric wires displayed on the goggles.

\section{Conflicts of Interest}

The authors declare no conflicts of interest regarding the publication of this paper.

\section{References}

[1] Kitamura, T. (2002) Is Electricity Invisible? Journal of IEE-J, 122, 307-310.

[2] Kuwahara, Y., Matsumoto, D. and Ushimoto, T. (2018) Detection of Charged Conductor by Radar. Proceeding of CAMA2018, Sweden, 3-6 September 2008. https://doi.org/10.1109/CAMA.2018.8530535

[3] Nogi, K., Kuwahara, Y. and Ushimoto, T. (2019) Design of Non-Contact Detection of a Power Application and Visualization System. Proceeding of IEICE General Conference, Japan, 5 March 2019.

[4] Kuwahara, Y., Nogi, K. and Ushimoto, T. (2019) Design of Non-Contact Detection of a Charged Conductor and Visualization System. Proceeding of 2019 IEEE International Workshop on Electromagnetics, Qingdao, China, 18-20 September 2019. https://doi.org/10.1109/iWEM.2019.8887901

[5] Nogi, K., Kuwahara, Y. and Ushimoto, T. (2020) Design of Non-Contact Detection of a Charged Conductor and Visualization System. IEICE Techical Report, 119, 19-24.

[6] Roberts, S. and Everson, R. (2001) Independent Component Analysis: Principles and Practice. Cambridge University Press, UK. https://doi.org/10.1017/CBO9780511624148

[7] Zhang, Z. (1998) A Frexible New Technique for Camera Calibration. Microsoft Research, USA.

[8] Analog Devices (2017) EV-RADAR-MMIC2 User Guide, Datasheet, UG-886, Rev. B.

[9] Teshirogi, T., Tanaka, M. and Chujo, W. (1985) Wideband Circularly Polarized Array with Sequential Rotation. Proceedings of ISAP, Tokyo, 117-120.

[10] Rahmat-Samii, Y. and Michielssen, E. (1999) Electromagnetic Optimization by Genetic Algorithms. Wiley-Interscience Publication, Wiley, New York.

[11] Andrew, R., Conn, Nicholas, I.M. Gould and Philippe L. (2000) Trust Region Methods. Society for Industrial and Applied Mathematics, Philadelphia.

[12] Hyvarinen, A. (1999) Fast and Robust Fixed-Point Algorithms for Independent Component analysis. IEEE Transaction on Neural Networks, 10, 626-634.

https://doi.org/10.1109/72.761722

[13] Japanese Patent No. 6696639, 2020. 